\title{
Utilisation Des Caractères Biochimiques, Agronomiques Et Morphologiques Pour L'évaluation De La Diversité Génétique Des Variants D'orangers Dans La Région Du Gharb, Maroc
}

\author{
Yacoubi R. \\ Institut National de Recherche Agronomique, INRA Kenitra Maroc. \\ Département de Biologie Université Ibn Tofail, \\ Faculté de Sciences, Kenitra, Maroc
}

N. Handaji

N. Arsalane

Institut National de Recherche Agronomique,

INRA Kenitra Maroc

M. Ibriz

Département de Biologie, Université Ibn Tofail,

Faculté de Sciences, Kenitra, Maroc

\section{H. Benyahia}

Institut National de Recherche Agronomique,

INRA Kenitra Maroc

Doi: 10.19044/esj.2018.v14n9p127 URL:http://dx.doi.org/10.19044/esj.2018.v14n9p127

\begin{abstract}
20 clones of orange trees, identified at the level of the collection of nucellar citrus varieties, have been the subject of several tests of behavior at the level of different citrus fruit sites in Morocco. Indeed, the present work aims to evaluate these variants on a clay soil at the Gharb region (INRA/Kenitra). The variants grafted on Citrange troyer were divided into three groups. Each group contains variants, genetically similar, from seedlings of the same parent variety. Clonal selection is based on the synthesis of the results of the study of the variability of the criteria of fruit quality and production. The average yield varies from $44.25 \mathrm{~kg} /$ tree to $217.30 \mathrm{~kg} /$ tree. While the average weight of a fruit varies from $95 \mathrm{~g}$ to $360 \mathrm{~g}$. The results showed a predominance of juicy and medium-sized fruits with an equatorial diameter greater than $56 \mathrm{~mm}$, acceptable for the fresh fruit market or the juice industry. The maturity coefficient study of the variants within each group, or between the three groups, showed that the harvest period extends over four months, from December to March. The final selection was focused on six
\end{abstract}


productive and good quality variants. These promising variants have been described according to UPOV, and then presented for registration in the official catalog.

Keywords: Citrus fruits, Selection, Variants, Quality, Production

\section{Résumé}

20 clones d'orangers, repérés au niveau de la collection des variétés nucellaires d'agrumes, ont fait l'objet de plusieurs essais de comportement au niveau des différents sites agrumicoles au Maroc. En effet, le présent travail vise l'évaluation de ces variants sur un sol argileux au niveau la région du Gharb (INRA /Kénitra). Les variants greffés sur le Citrange troyer ont été répartis en trois groupes. Chaque groupe contient les variants, génétiquement proches, issus des semis de la même variété mère. La sélection clonale est basée sur la synthèse des résultats de l'étude de la variabilité des critères de la qualité des fruits et de la production. Le rendement moyen varie de $44.25 \mathrm{Kg}$ /arbre à $217.30 \mathrm{~kg} /$ arbre. Tandis que le poids moyen d'un fruit varie de $95 \mathrm{~g}$ à $360 \mathrm{~g}$. Les résultats ont montré une prédominance des fruits juteux et de calibre moyen avec un diamètre équatorial supérieur de $56 \mathrm{~mm}$, acceptable pour le marché des fruits frais ou l'industrie du jus. L'étude de coefficient de maturité des variants au sein de chaque groupe, ou entre les trois groupes, a montré que la période de récolte s'étend sur quatre mois, de Décembre à Mars. La sélection finale a été portée sur six variants productifs et de bonne qualité. Ces variants prometteurs ont été décrits selon l'UPOV, et ensuite présentés pour l'inscription au catalogue officiel.

Mot clés : Agrumes, Sélection, Variants, Qualité, Production

\section{Introduction}

La culture des agrumes représente un intérêt économique de premier ordre en occupant ainsi la première place des productions fruitières mondiales. Le bassin méditerranéen est considéré comme la seconde zone de diversification des agrumes et a constitué un tremplin pour l'expansion des espèces agrumicoles cultivées à travers le monde. La diversité des variétés d'agrumes est souvent le résultat de modifications ou de croisements dus au hasard. Ces variétés sont apparues spontanément et se sont maintenues et propagées grâce à la capacité de reproduction non sexuée (apomixie) (Loussert, 1989). Chez ces espèces, l'apomixie se manifeste par le développement dans la graine d'embryons supplémentaires (polyembryonie) à celui issu de la fécondation. Ces embryons, provenant de cellules non reproductrices, ont tous la même constitution génétique et reproduisent à l'identique les caractères morphologiques de l'arbre initial. Ainsi, les 
variations de couleur, de forme et de goût du fruit des variétés d'oranges, de citrons, de pomelos sont quasiment toutes issues de modifications naturelles (mutations) des gènes impliqués dans l'expression de ces caractères. La sélection concerne donc ou bien les mutations spontanées identifiées en verger, ou les génotypes obtenus par hybridation, la mutagenèse induite, ou encore après avoir eu recours aux différentes approches biotechnologiques (hybridation somatique, transformation) (Ollitrault et Navarro, 2012). Par ailleurs, les orangers proches des mandariniers ont clairement introgressé des fragments du génome nucléaire de pamplemoussier dont l'implication comme parent femelle est démontrée par l'analyse du génome chloroplastique (Green et al., 1986 ; Nicolosi et al, 2000). Une étude récente a fourni une nouvelle idée sur l'origine de l'oranger $(C$. Sinensis), contredisant l'hypothèse attestant que $C$. sinensis est un hybride interspécifique direct entre un pamplemoussier (C. maxima) et un mandarinier (C. reticulata) (Nicolosi et al, 2000), soit qu'il est issu de rétrocroisement $\quad(C$. maxima $x$ C. reticulata) x $C$. reticulata) (Athani et al, 2009 ; Garcia-Lor et al., 2013) entre les pools de gènes $C$. maxima et $C$. Reticulata (Garcia-Lor et al., 2013). De plus, la polyembryonie permet d'obtenir des jeunes plants, exempts de virus similaires, au plant-mère et également de sélectionner des mutants améliorés qui peuvent être obtenus par graine. L'amélioration des agrumes par voie de sélection nucellaire prend beaucoup de temps en raison de la phase juvénile très longue et du nombre important d'épines. L'attente est souvent récompensée avec l'isolement de certains jeunes plants plus performants que le plant-mère sur le plan du rendement et qualité des fruits (Nicotra, 2001). Plusieurs variants nucellaires performants ont été obtenus, tels que les variants de la variété Valencia late (Valencia Cutter, Frost, Olinda, Delicia et Lue Gim Gong (Papadakis et al, 2008). La mandarine marocaine Nadorcott, obtenue également à partir de semis des graines de la variété Murcott honney, est très appréciée au niveau du marché d'exportation (Nadori et Nhami, 2005 ; Handaji et al, 2005). De même, des variants issus de semis apomictique de trois variétés d'orangers de l'espèce Citrus Sinensis (L) Obsbeck (Sanguinilli, Grosse sanguine, Salustiana) ont fait l'objet de plusieurs essais de comportement (Handaji et al, 2007 et 2013). Leur comportement dans les conditions pédoclimatiques du Gharb sur un sol argileux a fait 1'objet de la présente étude.

\section{Matériel et méthodes \\ Matériel végétal}

Le matériel végétal, objet de notre expérimentation, est constitué de $\mathbf{2 0}$ variants issus de semis apomictique, de trois variétés d'orangers de l'espèce Citrus Sinensis (L) Obsbeck (Sangunilli, Grosse Sanguine, Salustiana). Ils ont été préalablement repérés au niveau de la collection d'agrumes d'Afourer (Beni mellal). Trois groupes ont été mis en évidence et chaque groupe contient 
des variants issus de la même variété mère. Le nom provisoire de chaque variant porte le nom de la plante mère suivi par la lettre $\mathrm{V}$ et un numéro, comme Sangunilli V1 (Tableau 1). Ces variants d'orangers, installés en 1996 au domaine expérimental Sidi Allal Tazi, ont fait l'objet d'un essai de comportement variétal. Les arbres greffés sur Citrange troyer ont été plantés à un écartement de $6 m x 6 m$.

Tableau 1 : liste des variants d'orangers plantés au domaine expérimental de l'INRA (Sidi Allal Tazi)

\begin{tabular}{|c|c|c|c|}
\hline Variétés mères & Sanguinilli & Grosse Sanguine & Salustiana \\
\hline \multirow{6}{*}{ Variants } & V7 & V17 & V28 \\
& V8 & V18 & V29 \\
& V10 & V19 & V30 \\
& V11 & V20 & V31 \\
& V12 & V21 & V32 \\
& V13 & V22 & V33 \\
\hline Nombre & 7 & V23 & 6 \\
\hline
\end{tabular}

\section{Caractéristiques du site expérimental}

L'expérimentation est conduite au niveau du domaine Sidi Allal Tazi. Il s'étend sur une superficie de 200 ha dont 167 ha représentent la surface agricole utile (S.A.U). La superficie irriguée est de l'ordre de 70 ha avec une altitude approximative de $10,5 \mathrm{~m}$. Les sols du domaine expérimental sont de deux types «Dehs » léger représentant $80 \%$ et le «Dehs » lourd 20\%. Le climat est caractérisé par une pluviométrie moyenne annuelle de l'ordre de 400 à $700 \mathrm{~mm}$ et des températures moyennes de $4-7^{\circ} \mathrm{c}$ (minima), et de $32-34^{\circ}$ (maxima). Le nombre de journées gélives varie de 2 à 10 selon les années.

\section{Critères de qualité du jus du fruit}

Les paramètres étudiés sont liés, d'une part, à la qualité du fruit, et d'autre part au rendement en kg par arbre et à ses composantes. Au stade de maturité, des échantillons de 10 fruits ont été prélevés durant chaque année pour les analyses du jus.

Teneur en jus : L'extraction du jus a été effectuée par un extracteur à toupie tournante. Le jus recueilli est filtré à travers un filtre en plastique puis pesé. La teneur minimale en jus des orangers est fixée à $35 \%$ par l'Etablissement Autonome de Contrôle et de Coordination des Exportations (EACCE, 2010). La teneur en jus, exprimée en pourcentage massique, est donnée par la formule suivante :

Teneur en jus $=$ (poids de jus extrait de fruits $) \times 100 /$ poids total de ces fruits

Teneur en sucre : Elle a été déterminée par un réfractomètre à main dont les avantages sont bien connus : la précision, la rapidité de la mesure, la 
possibilité d'opérer avec une faible quantité de jus. Une goutte de jus déposée sur le prisme permet la lecture de l'extrait sec soluble. En fait, sa détermination est basée sur la capacité des sucres d'un jus à faire dévier la lumière. La teneur minimale en sucre des orangers est fixée à 9,5\% (EACCE, 2010).

Dosage de l'acidité : Pour le titrage de l'acidité du jus, $10 \mathrm{ml}$ de jus décanté a été utilisé et auquel trois gouttes de phénophtaléine sont ajoutées. La solution de $\mathrm{NaOH}(0,1 \mathrm{M})$, mise dans une burette de Mohr est versée lentement pour réaliser la titration.

Pendant le titrage, il est important d'utiliser un agitateur magnétique, pour homogénéiser continuellement la solution dans le bécher. Cette opération est essentielle, en particulier lorsque la solution s'approche à la neutralité, la fin du titrage est atteinte lorsque la couleur du jus devient rosâtre et persiste. La normalisation de l'acidité varie de 0.9 à $1.5 \%$ (EACCE, 2010).

Coefficient de maturité E/A : Le rapport E/A est un critère très utilisé dans la détermination de la date de cueillette et d'export des fruits d'agrumes. Il s'agit d'une augmentation de la teneur en sucre et d'une diminution de l'acidité. Le coefficient de maturité est déterminé par le rapport :

\section{Coefficient de Maturité= extrait sec soluble de sucre (E) /acidité du jus (en \%) (A)}

\section{Caractères agronomiques et morphologiques}

Les caractères morphologiques mesurés ont porté sur un échantillon de 30 fruits par variant et par an.

Poids moyen d'un fruit (g): Cette moyenne est déterminée en mesurant le poids total engendré par 10 fruits à l'aide d'une balance électrique

Rendement moyen (kg/arbre): Poids total de l'arbre en $\mathrm{kg}$. L'indice moyen d'alternance ABI (Alternate bearing index) est calculé sur le rendement moyen des arbres obtenus chaque année suivant la formule de Pearce et DobersekUrbanc (1997) :

$$
I=\frac{\sum \frac{\left|\mathrm{a}_{\mathrm{i}+1}-\mathrm{a}_{\mathrm{i}}\right|}{\mathrm{a}_{\mathrm{i}+1}+\mathrm{a}_{\mathrm{i}}}}{\mathrm{n}-1}
$$

a : Rendement des années correspondantes

$\mathrm{n}$ : Nombre d'années d'évaluation de rendement

L'indice de l'alternance varie de 0 à 1 . Si l'indice tend vers 0 , il y a une faible alternance ou vers 1 , une forte alternance.

Diamètre moyen $(\mathrm{mm})$ : Il est mesuré à l'aide d'un pied à coulisse électronique et gradué de 0 à $150 \mathrm{~mm}$, les diamètres équatorial (DE) et polaire (DP) sont exprimés en mm pour déterminer la forme du fruit (rapport $\mathrm{DE} / \mathrm{DP}=\mathrm{F}$; si $\mathrm{F} \geq 1$; le fruit aplatie alors si $\mathrm{F}<1$; le fruit ovale). 
Nombre de pépins par fruit: Le comptage des pépins a été réalisé manuellement

Epaisseur de l'écorce du fruit: L'écorce du fruit d'orange est constituée par le mésocarpe (albédo) et l'épicarpe (flavedo). L'épaisseur est mesurée en utilisant une règle graduée puis elle est classée en trois classes : mince $(\mathrm{E}<2 \mathrm{~mm})$, moyennement épaisse $(2 \mathrm{~mm}<\mathrm{E}<6 \mathrm{~mm})$ ou épaisse ( $\mathrm{E}>6 \mathrm{~mm})$. L'adhérence (forte, moyenne, légère) et la texture de la peau (lisse, rugueuse, semi rugueuse ou grêle).

Calibre des fruits : Il a été mesuré à l'aide d'un pied à coulisse. Le pourcentage de 3 catégories de calibre (1-4, 5-7, 8-11) a été déterminé.

-Analyses statistiques des données : les données obtenues ont été analysées à l'aide d'un logiciel de statistique SAS (Statistical Analysis System version 9.1 et version 5.5). L'analyse de la variance et la classification des moyennes ont été réalisées selon le test de Duncan avec une marge d'erreur de $5 \%$. De même, les données ont été traitées par les analyses de classification ou Cluster (UPGMA) et par l'indice de dissimilarité selon le logiciel DARWIN 5.0 .

\section{Résultats et discussion}

L'évaluation des performances des différents variants a montré des différences dans le rendement et la qualité des fruits.

\section{Mise à fruit et niveau de rendement}

Le rendement moyen est de $148 \mathrm{~kg} / \mathrm{arbre}$ et varie de $86 \mathrm{~kg} / \mathrm{arbre}$ à 216 $\mathrm{kg} / \mathrm{arbre}$. Les rendements obtenus ont différé selon les variants et les années d'étude (Tableau 2). L'analyse de la production annuelle de chaque groupe a montré que les trois groupes ont présenté une production relativement élevée pendant les années 2 et 3 . De même les variants du groupe Salustiana présentent les meilleurs rendements moyens pour les cinq années d'étude. Au sein du même groupe variants, le variant Sanguinilli V13, le variant Grosse sanguine V20 et le variant V31 ont donné relativement les meilleures moyennes de rendement, respectivement $139 \mathrm{~kg} / \mathrm{arbre}, 206 \mathrm{~kg} / \mathrm{arbre}$ et 252 $\mathrm{kg} /$ arbre. Par ailleurs, l'indice d'alternance a varié de 0.07 à 0.5 et aucune tendance vers l'alternance de la production n'a été constatée. La conduite du verger avec la pratique de la taille permet de réduire les effets de l'alternance. En effet, une taille annuelle sévère des arbres avec suppression jusqu'à $20 \%$ des branches favorise de rétablir l'équilibre entre la production des fruits et l'induction florale (Verreynne et Lovatt, 2009). Aussi, une nutrition équilibrée de l'arbre peut influencer l'indice d'alternance de manière significative, telle que l'application de l'urée, pendant l'hiver, ou de l'auxine peuvent augmenter la mise à fruit chaque année (Akhlaghi et Kangarshahi, 2011). Les variables environnementales, en particulier la température, affectent la croissance et le 
développement de la plante et aussi la productivité Kaleem et al, 2010). Le rendement dépend aussi du porte greffe utilisé (Abd El Motty et al., 2006 ; Bowman and McCollum , 2006 ; Bassal, 2009 ; Castle et al, 2010 ; Shafieizargar et al., 2012).

Etude de la variation intragroupe des critères de sélection avec comparaison entre les groupes variants sélectionnés et leurs plantes mères

L'étude statistique de la variabilité des critères de qualité du fruit selon les variants et leurs plantes mères sont résumés dans les tableaux 2, 3, 4 et 6 et selon les années dans la figure1.

\section{Variété Sanguinilli et ses variants}

La valeur moyenne du poids du fruit est de $145 \mathrm{~g}$. Elle varie de $124 \mathrm{~g}$ à $175 \mathrm{~g}$.

A cet effet, Les analyses statistiques ont montré qu'il n'existe pas un effet significatif entre la plante mère et ses variants pour tous les caractères étudiés à l'exception du poids moyen du fruit et la teneur en sucre (Tableau $3)$.

Ces mêmes analyses statistiques mettent en évidence trois groupes homogènes distincts : le groupe (a) inclus le variant V10 et la plante mère, le groupe (b) contenant le variant V7 et le groupe (ab) comprend les autres variants (V8, V11, V12, V13, V16). De même, la valeur moyenne du taux du sucre est de 10,77. Elle varie de 9,60 à 11,45. Statistiquement, il existe trois groupes : le groupe (a) contient le variant V7. Le groupe (b) comprend la plante mère (PM) et le groupe (ab) inclus les variants V8 V10 V11 V12 V13 et V16. Par ailleurs, le variant V11 est caractérisé par un taux de jus très élevé $(50 \%)$. Les analyses statistiques ont montré que l'effet de l'année est très significatif pour tous les caractères étudiés (Figure 1). Par ailleurs, Selon les années d'études, le nombre de pépins varie de 0 à 3 pépins en fonction des années. En général, le nombre de pépins par fruit serait aux facteurs climatiques. En effet, les basses températures pendant la floraison réduisent la capacité de germination du pollen et diminuent le nombre d'ovules susceptibles de devenir des graines (Pardo et al., 2010). Le pourcentage de jus le plus élevé a été obtenu la première annéet. Le poids du fruit varie d'une année à l'autre, cette variation est d'autant plus élevée que les années progressent, ici en l'occurrence, les années 2 et 3. La teneur en sucre varie selon le porte greffe, la variété, l'espèce et les conditions climatiques elle est élevée au stade de maturité. La valeur d'acidité la plus basse a été enregistrée en année 4. 
Tableau 2: Rendements moyens des variants d'orangers selon les années d'étude

\begin{tabular}{|c|c|c|c|c|c|c|c|}
\hline \multicolumn{8}{|c|}{ Rendement moyen (Kg/arbre) par année } \\
\hline \multirow{2}{*}{$\begin{array}{c}\text { Variants } \\
\text { V7 }\end{array}$} & 1 & 2 & 3 & 4 & 5 & Moyenne & $\begin{array}{c}\text { Indice } \\
\text { d'alternance }\end{array}$ \\
\hline & 50,00 & 155,00 & 62,50 & 86,75 & 25,00 & 75,85 & 0,39 \\
\hline V8 & 90,00 & 210,00 & 175,00 & 98,75 & 75,00 & 129,75 & 0,20 \\
\hline V10 & 90,00 & 255,00 & 75,00 & 112,50 & 143,75 & 135,25 & 0,33 \\
\hline V11 & 62,50 & 232,50 & 143,75 & 72,75 & 106,25 & 123,55 & 0,26 \\
\hline V12 & 80,00 & 207,50 & 125,00 & 88,25 & 111,50 & 122,45 & 0,19 \\
\hline V13 & 95,00 & 260,00 & 131,25 & 135,00 & 75,00 & 139,25 & 0,16 \\
\hline V16 & 45,00 & 167,50 & 183,33 & 126,50 & 81,25 & 120,72 & 0,20 \\
\hline Moyenne & 73,21 & 212,50 & 127,98 & 102,93 & 88,25 & 120,97 & 0,25 \\
\hline Sanguinilli & 62,50 & 102,50 & 100,75 & 70,75 & 96,25 & 86,55 & 0.22 \\
\hline V17 & 77,50 & 130,20 & 177,50 & 145,00 & 106,25 & 127,29 & 0,08 \\
\hline V18 & 65,00 & 137,50 & 172,50 & 146,75 & 100,00 & 124,35 & 0,27 \\
\hline V19 & 75,00 & 190,00 & 167,50 & 96,25 & 100,00 & 125,75 & 0,15 \\
\hline V20 & 120,00 & 307,50 & 287,50 & 130,00 & 187,50 & 206,50 & 0,20 \\
\hline V21 & 90,00 & 175,00 & 150,00 & 162,50 & 106,25 & 136,75 & 0,21 \\
\hline $\mathrm{V} 22$ & 85,00 & 210,00 & 150,00 & 224,50 & 105,00 & 154,90 & 0,22 \\
\hline V23 & 100,00 & 200,00 & 225,00 & 133,75 & 112,75 & 154,30 & 0,14 \\
\hline Moyenne & 87,50 & 192,89 & 190,00 & 148,39 & 116,82 & 147,12 & 0,18 \\
\hline $\begin{array}{c}\text { Grosse } \\
\text { sanguine }\end{array}$ & 45,00 & 112,23 & 118,75 & 119,00 & 200,00 & 120,69 & 0,50 \\
\hline V28 & 176,50 & 297,50 & 273,75 & 187,50 & 156,25 & 218,30 & 0,11 \\
\hline V29 & 100,00 & 200,00 & 202,50 & 200,00 & 205,00 & 181,50 & 0,07 \\
\hline V30 & 150,00 & 263,33 & 197,50 & 197,50 & 143,75 & 190,42 & 0,06 \\
\hline V31 & 105,00 & 375,50 & 250,00 & 171,25 & 362,50 & 252,85 & 0,33 \\
\hline V32 & 40,00 & 85,00 & 222,50 & 75,00 & 243,75 & 145,31 & 0,33 \\
\hline V33 & 80,00 & 175,00 & 62,50 & 37,25 & 56,25 & 82,20 & 0,34 \\
\hline Moyenne & 108,58 & 262,27 & 201,46 & 144,75 & 194,58 & 178,43 & 0,21 \\
\hline Salustiana & 145,00 & 282,50 & 325,00 & 138,00 & 263,75 & 252,31 & 0,40 \\
\hline Moye & 86,13 & 216,74 & 173,02 & 128,91 & 137,03 & 148,20 & 0,23 \\
\hline
\end{tabular}

Tableau 3: Etude statistique de la qualité du jus des variants de Sanguinilli

\begin{tabular}{|c|c|c|c|c|c|c|c|}
\hline Variants & PMF (g) & NPF & $\%$ jus & Acidité & E & E/A & DE/DP \\
\hline V7 & $124,00 \mathrm{~b}$ & $1,82 \mathrm{a}$ & $45,39 \mathrm{a}$ & $1,26 \mathrm{a}$ & $11,45 \mathrm{a}$ & $11,18 \mathrm{a}$ & $0,93 \mathrm{a}$ \\
\hline
\end{tabular}




\begin{tabular}{|c|c|c|c|c|c|c|c|}
\hline V8 & $144,25 \mathrm{ab}$ & $1,17 \mathrm{a}$ & $45,92 \mathrm{a}$ & $1,14 \mathrm{a}$ & $10,97 \mathrm{ab}$ & $10,89 \mathrm{a}$ & $0,90 \mathrm{a}$ \\
\hline V10 & $175,00 \mathrm{a}$ & $1,42 \mathrm{a}$ & $42,83 \mathrm{a}$ & $1,04 \mathrm{a}$ & $10,07 \mathrm{ab}$ & $9,87 \mathrm{a}$ & $1,05 \mathrm{a}$ \\
\hline $\mathrm{V} 11$ & $145,00 \mathrm{ab}$ & $2,1 \mathrm{a}$ & $50,02 \mathrm{a}$ & $1,08 \mathrm{a}$ & $10,97 \mathrm{ab}$ & 11,55 & $0,91 \mathrm{a}$ \\
\hline $\mathrm{V} 12$ & $133,75 \mathrm{ab}$ & $1,45 \mathrm{a}$ & $45,78 \mathrm{a}$ & $1,21 \mathrm{a}$ & $10,45 \mathrm{ab}$ & $9,75 \mathrm{a}$ & $0,93 \mathrm{a}$ \\
\hline $\mathrm{V} 13$ & $145,00 \mathrm{ab}$ & $2,12 \mathrm{a}$ & $43,99 \mathrm{a}$ & $1,07 \mathrm{a}$ & $10,50 \mathrm{ab}$ & $13,21 \mathrm{a}$ & $0,83 \mathrm{a}$ \\
\hline $\mathrm{V} 16$ & $151,50 \mathrm{ab}$ & $1,90 \mathrm{a}$ & $45,90 \mathrm{a}$ & $1,30 \mathrm{a}$ & $11,00 \mathrm{ab}$ & $9,23 \mathrm{a}$ & $0,92 \mathrm{a}$ \\
\hline PM1 & $171,25 \mathrm{a}$ & $0,67 \mathrm{a}$ & $42,42 \mathrm{a}$ & $1,22 \mathrm{a}$ & $9,60 \mathrm{~b}$ & $8,02 \mathrm{a}$ & $0,92 \mathrm{a}$ \\
\hline Moyenne & 145,50 & 1,710 & 45,69 & 1,16 & 10,77 & 11,08 & 0,94 \\
\hline Dunnett & $\mathrm{S}$ & $\mathrm{NS}$ & $\mathrm{NS}$ & $\mathrm{NS}$ & $\mathrm{S}$ & $\mathrm{NS}$ & $\mathrm{NS}$ \\
\hline CV\% & 18.53 & 14.2 & 12.01 & 21.63 & 8.12 & 25.36 & 4.13 \\
\hline
\end{tabular}

PM1 : Variété Sanguinilli

Tableau 4 : Etude statistique de la qualité du jus des variants de Gross sanguine

\begin{tabular}{|c|c|c|c|c|c|c|c|}
\hline Variants & PMF $(\mathrm{g})$ & NPF & $\%$ jus & A & E & E/A & DE/DP \\
\hline V17 & $188,75 \mathrm{a}$ & $0,67 \mathrm{a}$ & $39,73 \mathrm{a}$ & $1,46 \mathrm{a}$ & $11,10 \mathrm{ab}$ & $7,82 \mathrm{a}$ & $0,90 \mathrm{a}$ \\
\hline $\mathrm{V} 18$ & $180,00 \mathrm{a}$ & $0,57 \mathrm{a}$ & $40,87 \mathrm{a}$ & $1,28 \mathrm{a}$ & $11,04 \mathrm{ab}$ & $8,96 \mathrm{a}$ & $0,95 \mathrm{a}$ \\
\hline $\mathrm{V} 19$ & $176,67 \mathrm{a}$ & $0,63 \mathrm{a}$ & $42,67 \mathrm{a}$ & $1,33 \mathrm{a}$ & $11,06 \mathrm{ab}$ & $8,53 \mathrm{a}$ & $0,95 \mathrm{a}$ \\
\hline V20 & $210,00 \mathrm{a}$ & $0,37 \mathrm{a}$ & $42,48 \mathrm{a}$ & $1,39 \mathrm{a}$ & $11,87 \mathrm{a}$ & $8,61 \mathrm{a}$ & $0,96 \mathrm{a}$ \\
\hline V21 & $201,25 \mathrm{a}$ & $0,20 \mathrm{a}$ & $42,50 \mathrm{a}$ & $1,31 \mathrm{a}$ & $11,75 \mathrm{a}$ & $9,09 \mathrm{a}$ & $0,98 \mathrm{a}$ \\
\hline V22 & $186,67 \mathrm{a}$ & $1,00 \mathrm{a}$ & $39,34 \mathrm{a}$ & $1,47 \mathrm{a}$ & $11,93 \mathrm{a}$ & $8,28 \mathrm{a}$ & $0,94 \mathrm{a}$ \\
\hline V23 & $206,50 \mathrm{a}$ & $0,80 \mathrm{a}$ & $42,05 \mathrm{a}$ & $1,59 \mathrm{a}$ & $11,20 \mathrm{ab}$ & $7,35 \mathrm{a}$ & $0,91 \mathrm{a}$ \\
\hline PM2 & $172,50 \mathrm{a}$ & $0,60 \mathrm{a}$ & $42,69 \mathrm{a}$ & $1,15 \mathrm{a}$ & $10,26 \mathrm{~b}$ & $9,66 \mathrm{a}$ & $0,95 \mathrm{a}$ \\
\hline Moyenne & 192,83 & 0,61 & 41,38 & 1,40 & 11,47 & 8,38 & 0,94 \\
\hline Dunnett & $\mathrm{NS}$ & $\mathrm{NS}$ & $\mathrm{NS}$ & $\mathrm{NS}$ & $\mathrm{S}$ & $\mathrm{NS}$ & $\mathrm{NS}$ \\
\hline CV\% & 10.86 & 16.68 & 6.81 & 17.55 & 6.95 & 21.52 & 3.45 \\
\hline
\end{tabular}

PM2 : Variété Gross sanguine

Tableau 5 : Etude statistique de la qualité du jus des variants de Salustiana

\begin{tabular}{|c|c|c|c|c|c|c|c|}
\hline Variants & PMF $(\mathrm{g})$ & $\mathrm{NPF}$ & $\%$ jus & $\mathrm{A}$ & $\mathrm{E}$ & $\mathrm{E} / \mathrm{A}$ & DE/DP \\
\hline V28 & $197,00 \mathrm{a}$ & $0,40 \mathrm{a}$ & $47,31 \mathrm{abc}$ & $1,07 \mathrm{a}$ & $11,15 \mathrm{a}$ & $10,68 \mathrm{a}$ & $1,16 \mathrm{a}$ \\
\hline V29 & $186,25 \mathrm{a}$ & $0,60 \mathrm{a}$ & $48,18 \mathrm{ab}$ & $1,11 \mathrm{a}$ & $10,18 \mathrm{a}$ & $9,55 \mathrm{a}$ & $1,00 \mathrm{a}$ \\
\hline V30 & $211,67 \mathrm{a}$ & $0,80 \mathrm{a}$ & $43,44 \mathrm{abc}$ & $1,01 \mathrm{a}$ & $10,13 \mathrm{a}$ & $10,37 \mathrm{a}$ & $1,06 \mathrm{a}$ \\
\hline V31 & $210,50 \mathrm{a}$ & $0,35 \mathrm{a}$ & $49,86 \mathrm{a}$ & $1,06 \mathrm{a}$ & $10,10 \mathrm{a}$ & $9,76 \mathrm{a}$ & $1,04 \mathrm{a}$ \\
\hline V32 & $188,33 \mathrm{a}$ & $0,70 \mathrm{a}$ & $48,15 \mathrm{abc}$ & $0,92 \mathrm{a}$ & $10,87 \mathrm{a}$ & $12,10 \mathrm{a}$ & $1,14 \mathrm{a}$ \\
\hline V33 & $202,75 \mathrm{a}$ & $0,40 \mathrm{a}$ & $42,37 \mathrm{bc}$ & $0,83 \mathrm{a}$ & $10,80 \mathrm{a}$ & $13,28 \mathrm{a}$ & $1,06 \mathrm{a}$ \\
\hline PM3 & $167,50 \mathrm{a}$ & $0,05 \mathrm{a}$ & $41,23 \mathrm{c}$ & $0,93 \mathrm{a}$ & $9,80 \mathrm{a}$ & $11,38 \mathrm{a}$ & $1,06 \mathrm{a}$ \\
\hline Moyenne & 194,38 & 0,45 & 45,79 & 0,99 & 10,43 & 11,02 & 1,08 \\
\hline Dunnett & $\mathrm{NS}$ & $\mathrm{NS}$ & $\mathrm{NS}$ & $\mathrm{NS}$ & $\mathrm{NS}$ & $\mathrm{S}$ & $\mathrm{NS}$ \\
\hline CV $(\%)$ & 13,23 & 10,78 & 12,56 & 14,11 & 5,96 & 28,13 & 4,56 \\
\hline
\end{tabular}

\section{PM3 : Variété Salustiana}

cv : coefficient de variation PMF : poids moyen de fruit (g) NPF: nombre de pépins par fruit $\mathrm{E}$ : teneur en sucre $\mathrm{A}$ : Acidité $\mathrm{E} / \mathrm{A}$ : coefficient de maturité $\mathrm{DE}$ : diamètre équatorial DP : Diamètre polaire Coefficient DE/DP (forme du fruit), Au sein de chaque colonne, les valeurs portant les mêmes lettres sont significativement indifférentes avec $P>5 \%, S$ : significativement différentes de la plante mère selon le test de Dunnett, NS : pas de différence avec la plante mère 


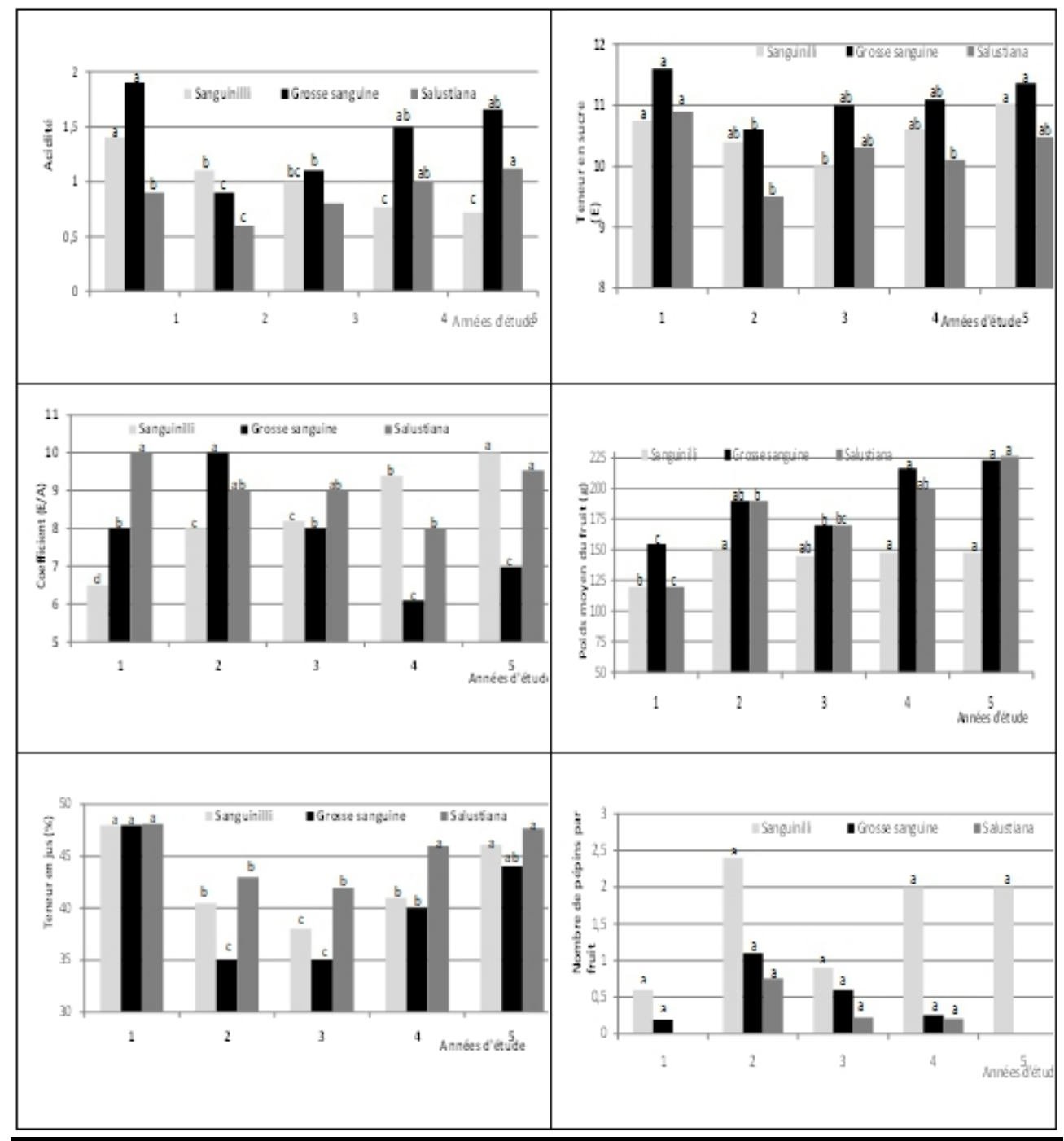

Figure 1: Evolution de la qualité du fruit des variants d'orangers selon les cinq années d'étude

(Au sein de chaque groupe des variants, les valeurs portant les mêmes lettres sont significativement indifférentes avec $\mathrm{P}>5$ )

\section{Variété Grosse Sanguine et ses variants}

Les analyses statistiques ont montré qu'il n'existe pas un effet significatif entre la plante mère et ses variants pour tous les caractères étudiés, à l'exception de la teneur en sucre (Tableau 4), La valeur moyenne est de 11. Elle varie entre 10,26 et 11,93. Statistiquement, il existe trois groupes : le groupe (a) inclut les variants les plus sucrés (V20, V21 et V22), le groupe (b) contenant la plante mère $(\mathrm{PM})$ et le groupe $(\mathrm{ab})$ comprend les variants V17, V18, V19, V23. Par ailleurs, selon les années, il existe une différence hautement significative pour tous les critères étudiés sauf le variable le nombre 
de pépins par fruit (Fig. 1), Il varie de 0,90 à 1,9 pour l'acidité ; de $35 \%$ à $48 \%$ (Teneur en jus) ; 0 à 1,1 (Nombre de pépins par fruit) ; 10,6 à 11,60 (Teneur en sucre) et de $155 \mathrm{~g}$ à $223 \mathrm{~g}$ (Poids moyen du fruit).

\section{Variété Salustiana et ses variants}

Les analyses statistiques ont monté qu'il n'existe pas un effet significatif entre la plante mère et ses variants pour tous les caractères étudiés, à l'exception du coefficient de la teneur en jus (Tableau 5), Statistiquement, il existe cinq groupes : le groupe (a) inclut le variant $1 \mathrm{~V} 31$, le groupe (ab) contient le variant V29, le groupe ( $a b c$ ) comprend V28, V30 et V32, le groupe bc V33 et en fin le groupe c renferme la plante mère. La valeur moyenne du nombre de pépins par fruit est de 0,47. Elle varie de 0,05 à 0,80 , Les fruits de tous les variants de Salustiana sont presque aspermes, Le variant V31 est caractérisé par les valeurs relativement élevé en poids moyen du fruit et la teneur en jus. En revanche, l'effet d'année est significatif pour tous les critères étudiés sauf le nombre de pépins par fruit. Il varie de 0,60 à 1,12 pour l'acidité ; de $42 \%$ à $48 \%$ (Teneur en jus); 0 à 0,75 (Nombre de pépins par fruit) ; 9,5 à 10,48 (Teneur en sucre) et de $120 \mathrm{~g}$ à $227 \mathrm{~g}$ (Poids moyen du fruit).

Par ailleurs, L'analyse statistique des variables de la qualité du fruit selon les années pour tous les clones d'orangers a montré que qu'il n'existe pas un effet significatif entre les années d'étude pour tous les variables à l'exception de la teneur en jus et le poids moyen du fruit (Tableau 6). Par conséquent, chaque génotype a un comportement différent selon les années.

L'étude de comportement de ces variants dans les autres sites ont montré qu'il existe une variabilité phénotypique entre les variants et la plante mère pour tous les critères étudiés (Handaji et al, 2007 et 2013). De même, des résultats similaires ont été signalés par (Mohar et al., 2011) qui ont montré que tous les caractères physico-chimiques de la maturité des fruits des orangers sont variables et que la variété Salustiana présente des valeurs maximales en poids moyen de fruits. De plus, le rendement et la qualité sont influencés par les conditions climatiques qui reposent essentiellement sur la température ayant sévi durant le cycle de vie des cultures (Killi et Altunbay, 2005 ; Dorji et Yap wattanaphun, 2011 ; Chelong et Sdoodé, 2012). De nombreuses Navel Orange ont été abandonnées à cause de stress hydrique survenu pendant la maturation des fruits (Kallsen et Sanden, 2011) confirmant ainsi le lien des conditions climatiques avec la qualité des agrumes rapportés par Reuther (1973) et Chelong and Sdoodee (2012).

Tableau 6 : Evolution de la qualité du fruit de tous les clones d'orangers étudiés selon les années cinq d'étude

\begin{tabular}{|c|c|c|c|c|c|c|}
\hline Années & acidité & \% jus & NPF & E & E/A & PMF (g) \\
\hline 1 & $1,40 \mathrm{a}$ & $48,03 \mathrm{a}$ & $0,26 \mathrm{a}$ & $11,08 \mathrm{a}$ & $8,17 \mathrm{a}$ & $131,67 \mathrm{c}$ \\
\hline 2 & $0,87 \mathrm{a}$ & $39,50 \mathrm{~b}$ & $1,42 \mathrm{a}$ & $10,17 \mathrm{a}$ & $9,00 \mathrm{a}$ & $176,67 \mathrm{ab}$ \\
\hline
\end{tabular}




\begin{tabular}{|c|c|c|c|c|c|c|}
\hline 3 & $0,97 \mathrm{a}$ & $38,33 \mathrm{~b}$ & $0,57 \mathrm{a}$ & $10,43 \mathrm{a}$ & $8,40 \mathrm{a}$ & $161,67 \mathrm{~b}$ \\
4 & $1,09 \mathrm{a}$ & $42,33 \mathrm{ab}$ & $0,82 \mathrm{a}$ & $10,60 \mathrm{a}$ & $8,23 \mathrm{a}$ & $188,14 \mathrm{a}$ \\
\hline 5 & $1,17 \mathrm{a}$ & $45,95 \mathrm{a}$ & $0,67 \mathrm{a}$ & $10,96 \mathrm{a}$ & $9,18 \mathrm{a}$ & $199,29 \mathrm{a}$ \\
\hline Moyenne & 1,10 & $42,83 \mathrm{ab}$ & $0,75 \mathrm{a}$ & $10,65 \mathrm{a}$ & $8,60 \mathrm{a}$ & $171,49 \mathrm{ab}$ \\
\hline
\end{tabular}

\section{Conclusion}

Sur la base des critères de qualité et de production, trois groupes de variants Salustiana, Sanguinilli et Grosse sanguine ont présenté des clones intéressants en terme de qualité et de production durant les années d'expérimentation. Ces clones sont principalement issus de la Sanguinilli (V11 et V13), la Grosse sanguine (V20 et V21) et Salustiana (V28, V31).

Ainsi, cette étude montre que les clones précités ont présenté des performances meilleures que les plantes mères et que, par conséquent, sont à valoriser dans les systèmes de production d'agrumes.

\section{References:}

1. Abd El Motty EZ, Shahin MFM, Hagagg LF (2006) Response of 'Valencia' orange trees budded on 'Troyer' citrange and Sour orange to foliar application of some macro and micronutrients, J Appl Sci Res 2: 952-965.

2. Akhlaghi Amiri N, Asadi Kangarshahi A. 2011. Evaluation the effects of auxin sucrose and nutrition on alternate bearing patte rs of Satsuma mandarin (citrus unshiu). Plant Production Research Journal. 17(3): 39-52, Gorgan university publication (in Persian).

3. Alireza Shafieizargar, Yahya Awang, Abdul Shukor Juraimi, Radziah Othman, 2012. Yield and fruit quality of Queen Orange [Citrus sinensis (L) Osb.] grafted on different rootstocks in Iran. Australian Journal of Crop Science. 6 ( 5): 777-783.

4. Athani, S, I,; Revanappa; Allolli, T, B, 2009, Variability of physical characters, quality and yield of kagzi lime (Citrus aurantifolia), Journal of Ecobiology Vol,25 No,3 pp,259-262 ref,6.

5. Bassal MA (2009) Growth, yield and fruit quality of 'Marisol' clementine grown on four rootstocks in Egypt, Sci Hortic 119: 132137.

6. Bowman KD, McCollum G, 2006, Performance of 'Hamlin' orange trees on 14 rootstocks in central Florida, Proc Fla State Hort Soc 119: 124-127.

7. Castle WS, Baldwin JC, Muraro RP (2010) Performance of 'Valencia' sweet orange trees on 12 rootstocks at two locations and an economic interpretation as a basis for rootstock selection, HortScience 45: 523533. 
8. Chellong I., Sdoodee S., 2012, Pollen viability, pollen germination and pollen tube growth of shogun (Citrus reticulate Blanco) under climate variability in southern Thailand. J. Agric. Technol. 8: 2297-2307.

9. Etablissement Autonome de Contrôle et de Coordination des Exportations, 2010, revue trimestrielle- Campagne 2010-2011 - No 30. http://web2.eacce.org.ma/Portals/0/Regl543Norme\%20Agrumes.pdf.

10. Garcia-Lor A., Curk F., Morillon R., Snoussi H., Ancillo G., Luro F., Navarro L., Ollitrault P. 2013. A nuclear phylogenetic analysis; SNPs, indels and SSRs deliver new insights into the relationships in the "true citrus fruit trees" group (Citrinae, Rutaceae) and the origin of cultivated species. Annals of Botany 111: 1-19.

11. Green RM, Vardi A Galum E, 1986, The Plastone of Citrus ; physical Map, variation Among Citrus Cultivars And Spacies, And comparison With Related Genera Theor, Appl, Genet, 72:761-769.

12. Handaji N., Benyahia H., Arsalane N., Ben Azouz A., Gaboun F., 2013, Evaluation pomologique et organoleptique de 34 variants d'orangers (Citrus sinensis (L.) Osbeck) issus de semis apomictique en essai dans la région du Gharb, "Revue de la recherche agronomique marocaine." Al Awamia, 127, 47-70.

13. Handaji N, Ait Haddou M, Ben Azouz A, Kabbage T, Srairi I, Arsalane N, Ben Yahia H, Essagide A, Gaboun F, 2007. Caractérisation et comportement de 35 variants d'agrumes dans la région de Souss Massa. Al Awamia ; 121-122: 110-134.

14. Handaji N, Arsalane N, Lamarti A, Dambier D, Benyahia H, Miaghizo $\mathrm{H}$, Cheikh OY, Ollitrault $\mathrm{P}, 2005$. Induction de l'embryogenèse somatique et régénération des plantules chez les mandariniers (Citrus reticulata L). El Awamia ; 114: 2 (2): 109-118.

15. Kaleem S, Hassan FU, Razzaq A (2010a). Growth rhythms in sunflower (Helianthus annuusL.) in response to environmental disparity. Afr. J. Biotechnol. 9:2442-2251.

16. Kallsen, C.E. and B. Sanden. 2011. Early Navel orange fruit yield, quality, and maturity in response to late-season water stress. Hortic. Sci., 46: 1163-1169.

17. Killi F., Altunbay S.G., 2005. Seed yield, oil content and yield components of confection and oilseed sunflower (Helianthus annuus L.) cultivars planted in different dates. Int. J. Agri. Biol. 7(1):21-24.

18. Loussert R., 1989. Techniques agricoles méditerranéennes, les agrumes, Vol. 1 :03-41.

19. Mohar TA, Abbas MM, Awan MZ, Javed MA, Aamer Farooq, 2011. Performance of different sweet orange varieties under Faisalabad 
conditions. Journal of Agricultural Research (Lahore). 49 (3): 363367.

20. Nadori et Nhami, 2005. la culture du Clementine au Maroc : évolution et perspectives.

21. Nicolosi E., Deng Z.N., Gentile A., La Malfa S., Continella G., Tribulato E. (2000) : citrusphy-logeny and genetic origin of important species as investigated by molecular markers. Theoretical and Applied Genetics, 100: 1155-1166.

22. Nicotra A, 2001. Hybrides de type mandarine présentant un intérêt récent pour la consommation à l'état frais. Problèmes et moyens de contrôle. Symposium sur les agrumes Chine/FAO.

23. Papadakis IE, Protopapadakis EE, Therios IN, 2008. Yield and fruit quality of two late- maturing Valencia orange tree varieties as affected by harvest date. Fruits (Paris) 63 (6): 327-334.

24. Pardo J, Cano A, Bermejo A, Zaragoza S, 2010, Temperature, pollen viability and seed formation in citrus crops. Ediciones y Promociones L. A. V., Valencia, Spain, Levante Agricola, 49 (399) : 20-29.

25. Reuther, W. 1973. Climate and citrus behavior. In The Citrus Industry. 2nd Ed., Vol. 3, (ed.) W. Reuter. pp. 280-337. Berkeley: University of California Press.

26. Verreynne JS, Lovatt CJ, 2009. Influences Return Bloom in Alternate Bearing 'Pixie' Mandarin. SOC. HORT. SCI. 134(3) : 299-307. 


\section{Annexe1: Diamètre moyen du fruit des variants d'orangers et leurs plantes mères}

\begin{tabular}{|c|c|c|c|c|}
\hline \multirow{2}{*}{$\begin{array}{c}\text { Goupe de } \\
\text { variants }\end{array}$} & \multirow[b]{2}{*}{ Codes } & \multicolumn{3}{|c|}{ Diamètre moyen (mm) } \\
\hline & & Equatorial & Polaire & DE/DP \\
\hline \multirow{9}{*}{ Sanguinilli } & V7 & 55,81 & 60,01 & 0,93 \\
\hline & V8 & 61,84 & 68,71 & 0,9 \\
\hline & V10 & 73,3 & 69,81 & 1,05 \\
\hline & V11 & 67,26 & 73,91 & 0,91 \\
\hline & V12 & 61,51 & 66,14 & 0,93 \\
\hline & V13 & 57,36 & 69,11 & 0,83 \\
\hline & V16 & 67,54 & 73,41 & 0,92 \\
\hline & Moyenne & 63,52 & 69,04 & 0,92 \\
\hline & PM1 & 66,54 & 70,79 & 0,94 \\
\hline \multirow{8}{*}{ Grosse sanguine } & V17 & 69,12 & 76,80 & 0,9 \\
\hline & V18 & 74,68 & 78,61 & 0,95 \\
\hline & V19 & 72 & 75,79 & 0,95 \\
\hline & V20 & 75,5 & 78,65 & 0,96 \\
\hline & V21 & 74,36 & 75,88 & 0,98 \\
\hline & V23 & 73,75 & 81,04 & 0,91 \\
\hline & Moyenne & 73,23 & 77,90 & 0,94 \\
\hline & PM2 & 69,49 & 73,15 & 0,95 \\
\hline \multirow{8}{*}{ Salustiana } & V28 & 75,27 & 64,89 & 1,16 \\
\hline & V29 & 75,05 & 75,05 & 1 \\
\hline & V30 & 81,54 & 76,92 & 1,06 \\
\hline & V31 & 77,31 & 74,34 & 1,04 \\
\hline & V32 & 71,22 & 62,47 & 1,14 \\
\hline & V33 & 82,54 & 77,87 & 1,06 \\
\hline & Moyenne & 77,15 & 71,44 & 1,08 \\
\hline & PM3 & 71,15 & 67,12 & 1,06 \\
\hline
\end{tabular}



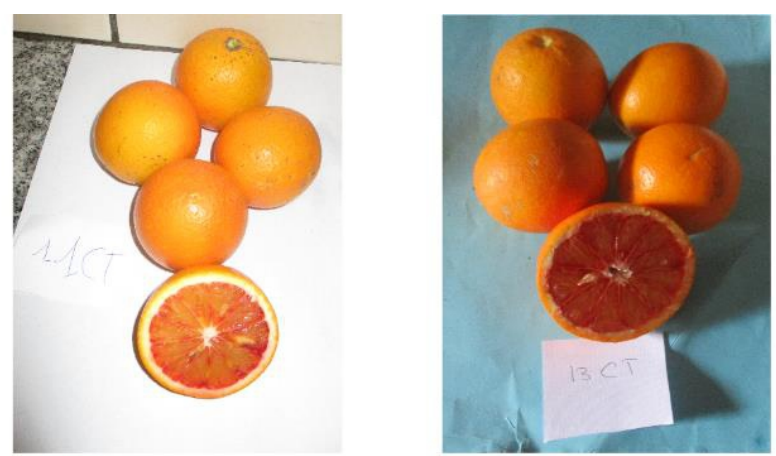

Sanguinilli
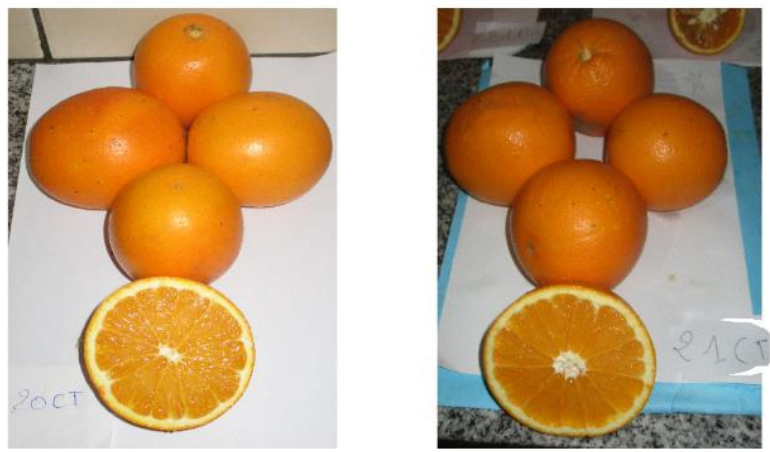

Grosse Sanguine
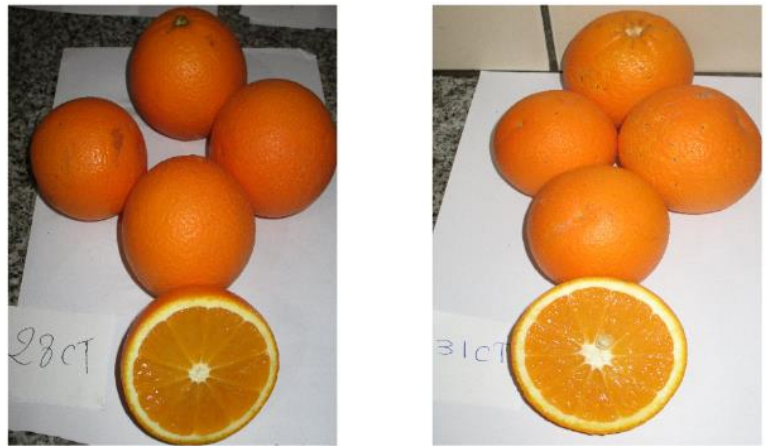

Salustiana

Figure 2 : Les Variants d'orangers sélectionnés 